\title{
The Metapopulation Dynamics of an Infectious Disease: Tuberculosis in Possums
}

\author{
G. R. Fulford ${ }^{1}$ and M. G. Roberts ${ }^{2}$ \\ AgResearch, Wallaceville Animal Research Centre, P.O. Box 40063, Upper Hutt, New Zealand
}

and

\author{
J. A. P. Heesterbeek ${ }^{3}$ \\ Centre for Biometry, P.O. Box 16, 6700 AA Wageningen, The Netherlands
}

Received September 15, 2000

\begin{abstract}
An SEI metapopulation model is developed for the spread of an infectious agent by migration. The model portrays two age classes on a number of patches connected by migration routes which are used as host animals mature. A feature of this model is that the basic reproduction ratio may be computed directly, using a scheme that separates topography, demography, and epidemiology. We also provide formulas for individual patch basic reproduction numbers and discuss their connection with the basic reproduction ratio for the system. The model is applied to the problem of spatial spread of bovine tuberculosis in a possum population. The temporal dynamics of infection are investigated for some generic networks of migration links, and the basic reproduction ratio is computed-its value is not greatly different from that for a homogeneous model. Three scenarios are considered for the control of bovine tuberculosis in possums where the spatial aspect is shown to be crucial for the design of disease management operations. $\odot 2002$ Elsevier Science (USA)
\end{abstract}

\section{INTRODUCTION}

Mathematical models have been used to help guide control policies for infectious diseases of humans and animals since the pioneering work of Kermack and McKendrick (see Anderson and May, 1991). In many models the subpopulations of susceptibles and infectives are assumed to be homogeneous, that is, each member of

${ }^{1}$ Current address: Urban Geoscience Division, Geoscience Australia, GPO Box 378, Canberra ACT, Australia. E-mail: glenn. fulford@ga.gov.au.

${ }^{2}$ E-mail: mick.roberts@agreasearch.co.nz.

${ }^{3}$ Current address: Quantitative Veterinary Epidemiology Group, Faculty of Veterinary Medicine, Yalelaan 7, 3584 CL Utrecht, The Netherlands. E-mail: j.a.p.heesterbeek@vet.uu.nl. the subpopulation is treated in exactly the same way. There has been a recognised need to incorporate spatial heterogeneity into epidemic models (Dobson and Grenfell, 1995), especially when explicit spatial information is required or when homogeneous models fail to adequately describe observed behaviour. Some of the issues arising in spatial modelling of diseases have been reviewed by Bolker (1995).

With spatial modelling it becomes important to account for the individual's behavioural characteristics with respect to space, e.g., mixing behaviour and migration. Several different approaches have been used (see the review by Mollison and Levin, 1995.) These include using reaction-diffusion equations (e.g., Murray, 1990; Louie et al., 1993; Pech and McIlroy, 1990), which is useful 
where individuals undergo random walks but is not realistic for animals which frequent a home range. Another approach uses the metapopulation concept (Hanski and Gilpin, 1997), which assumes homogeneous mixing in discrete patches with migration between patches. This is the approach developed here.

An important quantity in epidemiology is the basic reproduction ratio, usually denoted by $R_{0}$. It is a threshold quantity which determines whether the infectious agent persists or dies out, and therefore has useful application for the design of control strategies. It is easy to obtain a formula for $R_{0}$ in terms of the parameters of simple homogeneous models, but more difficult for models that have spatial heterogeneity. Lloyd and May (1996) achieved this using a model where heterogeneity was incorporated through the mixing of individuals from separate home patches, with no migration between patches. They obtained an explicit formula for $R_{0}$ as the sum of basic reproduction ratios for each patch. Jacquez et al. (1995) also considered various models with heterogeneous mixing and obtained threshold criteria. In this paper we apply the general method developed in Diekmann et al. (1990) for the calculation of $R_{0}$ in structured populations to the spread of an infectious disease in a wild animal metapopulation. The model is simple enough to enable the calculation of $R_{0}$ but has sufficient complexity to explore the effects of spatial heterogeneity due to migration.

The model developed in this paper is motivated by the spread of bovine tuberculosis (Mycobacterium bovis) in the common brushtail possum (Trichosurus vulpecula) in New Zealand. Possums are thought to be the main wildlife reservoir for the disease, which presents a threat to export industries. A similar problem exists in the United Kingdom and Ireland, with badgers being the main reservoir for tuberculosis (see, for example, Ruxton, 1996; Swinton et al., 1997), but there are differences between possums and badgers which have important implications for control (see Kao and Roberts, 1999).

Possums are nocturnal animals and are generally solitary. Breeding occurs throughout the year, but mainly in the autumn with a secondary pulse in the spring. Possums frequent a home range, typically 0.6-3.5 ha, Cowan (1990), on which they use several dens. There is debate on the principal method of transmission of tuberculosis between possums, with sequential sharing of den sites, transmission during mating, transmission between fighting males competing for mates, and transmission from mothers to pouch young being suggested, (Morris and Pfeiffer, 1995; Jackson et al., 1995).
However, Caley et al. (1998) report very low probabilities for simultaneous den sharing.

Mathematical models have been suggested to guide possum culling programs (see Roberts, 1996; Barlow, 1996), and while there has been some success there are areas where the programs have not worked. One explanation for this is that the models have not always been applicable to certain landscapes where the spatial properties vary significantly. Another possible reason is that maturing possums often migrate large distances. These are typically 1 - to 2-year-old males after their first winter as an independent juvenile. Exposed possums from an area where tuberculosis is endemic may therefore spread the infectious agent into the controlled area.

Some attempts at modelling the spatial aspect of the spread of tuberculosis among possums have been made using computer simulations, Pfeiffer (1994) used an individual-based Monte Carlo model, for example. While many aspects of possum biology and behaviour can be incorporated into this type of model, large numbers of parameters are required, many of which are not known with any accuracy; and since the model is stochastic many runs may be needed to generate useful statistics. Barlow $(1993,1994)$ developed a mixed deterministic/stochastic computer simulation model with disease dynamics applied to adjoining $1 \mathrm{~km}^{2}$ square grid patches. The basic reproduction ratio $R_{0}$ was not calculated, but the model did provide some insights into the differences with nonspatial models.

Our proposed model is simple enough to enable the calculation of $R_{0}$ but has sufficient complexity to explore the effects of spatial heterogeneity due to migration. We first compare a one-patch configuration with some simple two-patch configurations, and then we consider three scenarios for disease control based on a larger number of habitat patches.

\section{AN SEI MODEL FOR A METAPOPULATION}

Consider a metapopulation of $n$ populations of animals, each on a distinct patch of habitat, but with the patches connected by the migration of a proportion of juveniles upon maturity. Some individuals within the metapopulation are infected with a fatal infectious disease, which can be transmitted between hosts on the same patch by infectious contact, and (pseudo) vertically (infection of juveniles by their mothers), but can only be 
transmitted between patches by the migration of host animals that have been exposed to infection.

We consider two age-classes, adults and juveniles. We assume that only juveniles migrate and that they become adults on the destination patch, i.e., only those juveniles on the brink of adulthood migrate. Migrating juveniles consist of healthy and exposed individuals, but we assume that those who are infectious do not migrate. We assume births, deaths, and migration to be spread out continuously over time.

We number the patches $k=1, \ldots, n$, and use the convention that odd indices refer to juveniles and even indices refer to adults. Hence, the population density of susceptible, exposed and infectious juveniles on patch- $k$ is $S_{2 k-1}, E_{2 k-1}, I_{2 k-1}$, respectively, and the density of susceptible, exposed and infectious adults is $S_{2 k}, E_{2 k}, I_{2 k}$, respectively. The total host population density on patch- $k$ is $P_{k}=N_{2 k-1}+N_{2 k}$ with $N_{2 k-1}=S_{2 k-1}+E_{2 k-1}+I_{2 k-1}$ and $N_{2 k}=S_{2 k}+E_{2 k}+I_{2 k}$. The equations for the dynamics of the juvenile subpopulations are

$$
\begin{aligned}
\frac{d N_{2 k-1}}{d t}= & B_{k} N_{2 k}-\left(D_{2 k-1}+a\right) N_{2 k-1}-\alpha I_{2 k-1} \\
\frac{d E_{2 k-1}}{d t}= & \beta_{v} B_{k} I_{2 k}+\beta_{11} C_{11} S_{2 k-1} \frac{I_{2 k-1}}{N_{2 k-1}} \\
& +\beta_{12} C_{12} S_{2 k-1} \frac{I_{2 k}}{N_{2 k}}-\left(D_{2 k-1}+\sigma+a\right) E_{2 k-1} \\
\frac{d I_{2 k-1}}{d t}= & \sigma E_{2 k-1}-\left(D_{2 k-1}+\alpha+a\right) I_{2 k-1}
\end{aligned}
$$

and the equations for the dynamics of the adult subpopulations are

$$
\begin{aligned}
\frac{d N_{2 k}}{d t}= & a \sum_{l=1}^{n} m_{k l} N_{2 l-1}-D_{2 k} N_{2 k}-\alpha I_{2 k} \\
\frac{d E_{2 k}}{d t}= & a \sum_{l=1}^{n} m_{k l} E_{2 l-1}+\beta_{21} C_{21} S_{2 k} \frac{I_{2 k-1}}{N_{2 k-1}} \\
& +\beta_{22} C_{22} S_{2 k} \frac{I_{2 k}}{N_{2 k}}-\left(D_{2 k}+\sigma\right) E_{2 k} \\
\frac{d I_{2 k}}{d t}= & a I_{2 k-1}+\sigma E_{2 k}-\left(D_{2 k}+\alpha\right) I_{2 k} .
\end{aligned}
$$

The per-capita birth rate $B_{k}$ and the juvenile and adult per-capita death rates $D_{2 k-1}$ and $D_{2 k}$, respectively, are nonincreasing and nondecreasing functions of the local population densities, respectively. The parameter $a$ represents the rate at which juveniles mature. The func- tions $C_{11}, C_{12}, C_{21}, C_{22}$ are contact rate functions; for example, $C_{12}$ is the rate of adult contacts made by a single juvenile. Each of these are nondecreasing functions of the local adult and juvenile subpopulation densities. We do not assume the contact rate functions to be habitat dependent (although it would not be difficult to do so if it were needed). Other parameters are: $\sigma$, the reciprocal of the latent period, $\beta_{11}, \beta_{12}, \beta_{21}$, and $\beta_{22}$ the probabilities of transmission given contact between a susceptible and an infectious host (juvenile to juvenile, juvenile to adult, adult to juvenile, and adult to adult, respectively). The term $\beta_{v} B_{k} I_{2 k}$ in Eq. (2) accounts for (pseudo)vertical transmission. Given $B_{k}$ is the per-capita birth rate, then $2 B_{k}$ is the birth rate per female and $I_{2 k} / 2$ is the density of infectious females (assuming a 1:1 sex ratio), thus $\beta_{v}$ is the probability that an offspring of an infectious mother is infected.

We define $a m_{k l}$ to be the per-capita migration rate for a maturing juvenile from patch- $l$ that becomes an adult on patch- $k$. The terms $m_{k k}$ represent the probability that a juvenile remains on the patch on which it was born. That is, juveniles leave the $N_{2 k-1}$ and $E_{2 k-1}$ classes with rate $a$ and enter the $N_{2 k}$ and $E_{2 k}$ classes with rate $a m_{k k}$. The infectious agent is spread to other patches by maturing juveniles who are from the exposed classes $E_{2 l-1}$.

Now consider those that migrate from patch- $l$ to patch- $k$. In $\tau$ time units a total of $a N_{2 l-1} A_{l} \tau$ host animals mature on patch- $l$ where $A_{l}$ is the area of the patch. Let the probability of a juvenile leaving patch- $l$ be denoted by $z_{l}$ and let the fraction of those leaving patch- $l$ and successfully arriving on patch- $k$ be $s_{k l}$. Then, neglecting the time required for migration, the number of animals entering patch- $k$ from patch- $l$ over a time period of length $\tau$ is $s_{k l} z_{l} a N_{2 l-1} A_{l} \tau$ and the rate at which this increases the population density of patch- $k$ is $s_{k l} z_{l} a N_{2 l-1} A_{l} / A_{k}$. Hence

$$
m_{k l}=s_{k l} z_{l} \frac{A_{l}}{A_{k}} .
$$

\section{THE CALCULATION OF $\boldsymbol{R}_{0}$}

The basic reproduction ratio $\left(R_{0}\right)$ for an infectious agent is defined as the expected number of secondary infections arising over the infectious lifetime of a single primary infective in a population consisting entirely of susceptibles. It may be determined by constructing the next generation matrix based on infection-free equilibrium densities and computing its spectral radius (Diekmann et al., 1990, Heesterbeek and Roberts, 1995, 
Diekmann and Heesterbeek, 2000). If $R_{0}>1$ the infectious agent can persist in the (meta)population, but if $R_{0}<1$ it will be eliminated over time. We first calculate $R_{0}$ for the model on a single patch, then for two patches connected by juvenile migration and then generalise to any multi-patch system using three generic 4-patch structures as examples.

To calculate $R_{0}$ we first need to determine the infectionfree equilibrium. This is straightforward for a single patch model, but must be carried out numerically for multiple patches connected by migration. We assume uniqueness of this equilibrium but we have not seen any numerical evidence to the contrary.

\section{The Single-Patch Model}

Assume a unique, nonzero, infection-free steady-state ( $\left.N_{1}, N_{2}\right)$ exists. We calculate $R_{0}$ as follows. We first construct the next generation matrix, whose elements are the expected number of secondary cases within an infection class due to a single primary case within that class, given a completely susceptible population. Equivalently, we can consider secondary exposed hosts due to a primary exposed host, which is more straightforward (see Fig. 1).

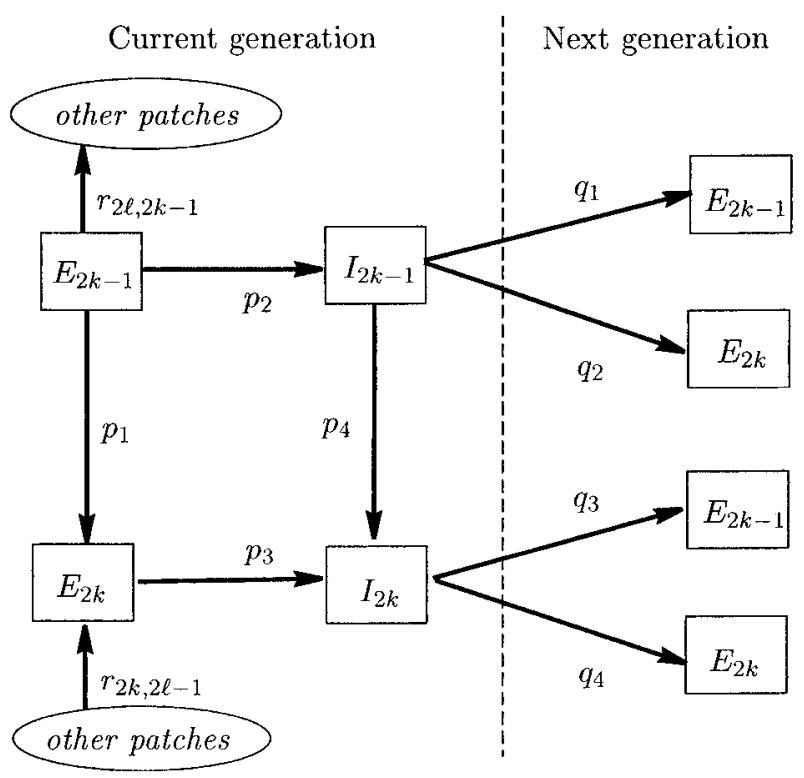

FIG. 1. The possible routes of infection within and between generations on a patch. The $p_{i}$ values are transition probabilities between age and infection classes on the same patch (patch-k) and in the same generation, and the $q_{i}$ values measure transmission of infection on the patch $(i=1 \ldots 4)$. The values $r_{2 l, 2 k-1}$ and $r_{2 k, 2 l-1}$ are the migration rates from patch- $k$ to patch- $l$, and in the opposite direction. The compartment labels $E_{2 k-1}, E_{2 k}$ are the densities of exposed juveniles and adults, respectively, on patch- $k$, and $I_{2 k-1}, I_{2 k}$ are the densities of infectious juveniles and adults, respectively.
We observe that each individual from the $E_{1}$ class joins the $E_{2}$ or $I_{1}$ classes with probability $p_{1}$ or $p_{2}$, respectively, and each individual in the $E_{2}$ or $I_{1}$ classes joins the $I_{2}$ class with probability $p_{3}$ or $p_{4}$, respectively, where

$$
\begin{array}{ll}
p_{1}=\frac{a m_{11}}{D_{1}+\sigma+a} & p_{2}=\frac{\sigma}{D_{1}+\sigma+a} \\
p_{3}=\frac{\sigma}{D_{2}+\sigma} & p_{4}=\frac{a}{D_{1}+\alpha+a} .
\end{array}
$$

If the 1-patch system is closed then $m_{11}=1$. The increases in density of the $E_{1}$ and $E_{2}$ classes due to a small density $\delta I_{1} \mathrm{ha}^{-1}$ of infectious juvenile possums throughout their infectious lifetimes are $q_{1} \delta I_{1}$ and $q_{2} \delta I_{1}$, respectively, and the increases due to a small density $\delta I_{2} \mathrm{ha}^{-1}$ of adult infectious are $q_{3} \delta I_{2}$ and $q_{4} \delta I_{2}$, respectively, where

$$
\begin{array}{ll}
q_{1}=\frac{\beta_{11} C_{11}}{D_{1}+\alpha+a} & q_{2}=\frac{\beta_{12} C_{12}}{D_{1}+\alpha+a} \\
q_{3}=\frac{1}{D_{2}+\alpha}\left(\beta_{v} B_{1}+\beta_{12} C_{12}\right) & q_{4}=\frac{\beta_{22} C_{22}}{D_{2}+\alpha} .
\end{array}
$$

We then calculate the increase in density of secondgeneration $E_{1}$ and $E_{2}$ classes due to each unit density of first-generation $E_{1}$ and $E_{2}$,

$$
\begin{aligned}
& \left(E_{1} \rightarrow E_{1}\right)=p_{2} q_{1}+\left(p_{1} p_{3}+p_{2} p_{4}\right) q_{3} \\
& \left(E_{1} \rightarrow E_{2}\right)=p_{2} q_{2}+\left(p_{1} p_{3}+p_{2} p_{4}\right) q_{4} \\
& \left(E_{2} \rightarrow E_{1}\right)=p_{3} q_{3} \\
& \left(E_{2} \rightarrow E_{2}\right)=p_{3} q_{4} .
\end{aligned}
$$

Finally, we calculate $R_{0}$ as the spectral radius of the next-generation matrix

$$
R_{0}=\rho\left(\begin{array}{ll}
\left(E_{1} \rightarrow E_{1}\right) & \left(E_{2} \rightarrow E_{1}\right) \\
\left(E_{1} \rightarrow E_{2}\right) & \left(E_{2} \rightarrow E_{2}\right)
\end{array}\right)
$$

where $\rho$ denotes the dominant eigenvalue (largest in magnitude).

\section{The Two-Patch Model}

Assuming the existence of a unique infection-free equilibrium $\left(N_{1}, N_{2}, N_{3}, N_{4}\right)$, to be determined numerically, we calculate $R_{0}$ in a way similar to the single patch model. For patch-1 the probabilities of transition from $E_{1}$ to $E_{2}, E_{1}$ to $I_{1}, E_{2}$ to $I_{2}$ and $I_{1}$ to $I_{2}$ are as for the single patch model. For patch-2 we define $p_{5}$ to $p_{8}$ similarly to 
$p_{1}$ to $p_{4}$, just replace $m_{11}$ with $m_{22}$ and $D_{1}$ and $D_{2}$ with $D_{3}$ and $D_{4}$, respectively. The parameters $q_{1}$ to $q_{4}$ are specified as for the single patch model, and on patch-2

$$
\begin{aligned}
q_{5} & =\frac{\beta_{11} C_{11}}{D_{3}+\alpha+a} & q_{6} & =\frac{\beta_{12} C_{12}}{D_{3}+\alpha+a} \\
q_{7} & =\frac{1}{D_{4}+\alpha}\left(\beta_{v} B_{2}+\beta_{12} C_{12}\right) & q_{8} & =\frac{\beta_{22} C_{22}}{D_{4}+\alpha} .
\end{aligned}
$$

The routes of infection within patch-2 are

$$
\begin{aligned}
& \left(E_{3} \rightarrow E_{3}\right)=p_{6} q_{5}+\left(p_{5} p_{7}+p_{6} p_{8}\right) q_{7} \\
& \left(E_{3} \rightarrow E_{4}\right)=p_{6} q_{6}+\left(p_{5} p_{7}+p_{6} p_{8}\right) q_{8} \\
& \left(E_{4} \rightarrow E_{3}\right)=p_{7} q_{7} \\
& \left(E_{4} \rightarrow E_{4}\right)=p_{7} q_{8} .
\end{aligned}
$$

Hence we calculate $R_{0}$ as the spectral radius of the next generation matrix

$$
R_{0}=\rho\left(\begin{array}{ll}
\mathrm{K}_{11} & \mathrm{~K}_{12} \\
\mathrm{~K}_{21} & \mathrm{~K}_{22}
\end{array}\right)
$$

where

$$
\mathrm{K}_{11}=\left(\begin{array}{ll}
\left(E_{1} \rightarrow E_{1}\right) & \left(E_{2} \rightarrow E_{1}\right) \\
\left(E_{1} \rightarrow E_{2}\right) & \left(E_{2} \rightarrow E_{2}\right)
\end{array}\right)
$$

is the next generation matrix for patch-1,

$$
\mathrm{K}_{22}=\left(\begin{array}{ll}
\left(E_{3} \rightarrow E_{3}\right) & \left(E_{4} \rightarrow E_{3}\right) \\
\left(E_{3} \rightarrow E_{4}\right) & \left(E_{4} \rightarrow E_{4}\right)
\end{array}\right)
$$

is the next generation matrix for patch-2, and the matrices $K_{12}$ and $K_{21}$ account for inter-patch migrations of juveniles that have been exposed to infection. Those from patch-2 (the $E_{3}$ class) migrate to patch-1, become infectious and give rise to increases in the $E_{1}$ or $E_{2}$ classes. The $E_{4}$ class, being adults, do not migrate. Hence

$$
\mathrm{K}_{12}=\left(\begin{array}{ll}
r_{23} p_{3} q_{3} & 0 \\
r_{23} p_{3} q_{4} & 0
\end{array}\right)
$$

and similarly

$$
\mathrm{K}_{21}=\left(\begin{array}{ll}
r_{41} p_{7} q_{7} & 0 \\
r_{41} p_{7} q_{8} & 0
\end{array}\right) .
$$

The parameters $r_{i j}$ are defined by

$$
r_{i j}=\frac{a m_{i j}}{D_{j}+\sigma+a} .
$$

For example, to calculate the element $\left(\mathrm{K}_{12}\right)_{11}$ we need to determine the average increase in the density of second generation exposed juvenile hosts on patch-1 (class $E_{1}$ ) due to a typical unit density of first generation exposed juveniles on patch-2 (class $E_{3}$ ). This term equals the product of:

$r_{23}$ the probability that a juvenile from patch-2 migrates to patch-1 after it has been exposed to infection, becoming an adult in the process, $\left(E_{3} \rightarrow E_{1}\right)$;

$p_{3}$ the probability that an exposed adult on patch-1 becomes infectious, $\left(E_{1} \rightarrow I_{1}\right)$; and

$q_{3}$ the average number of exposures to infection among juveniles due to a typical infectious adult on patch-1 $\left(I_{1} \rightarrow E_{1}\right)$.

\section{Multi-Patch Models}

We have now separated demography, epidemiology, and topography and provided a system for determining $R_{0}$ on any metapopulation structure. For $n$ patches the next generation matrix $\mathrm{M}$ is a $2 n \times 2 n$ matrix, that may by represented as an $n \times n$ matrix of $2 \times 2$ submatrices, $\mathbf{M}=\left(\mathrm{K}_{l k}\right)$. The diagonal submatrices are

$$
\mathrm{K}_{k k}=\left(\begin{array}{cc}
\left(E_{2 k-1} \rightarrow E_{2 k-1}\right) & \left(E_{2 k} \rightarrow E_{2 k-1}\right) \\
\left(E_{2 k-1} \rightarrow E_{2 k}\right) & \left(E_{2 k} \rightarrow E_{2 k}\right)
\end{array}\right)
$$

and the off-diagonal submatrices are

$$
\mathrm{K}_{l k}=\left(\begin{array}{cc}
\left(E_{2 k-1} \rightarrow E_{2 l-1}\right) & 0 \\
\left(E_{2 k-1} \rightarrow E_{2 l}\right) & 0
\end{array}\right) .
$$

The entries for these submatrices are calculated as in the previous sections, and $R_{0}=\rho(\mathrm{M})$. Where there is no migration between patches $k$ and $l$ the submatrix $\mathrm{K}_{l k}$ has zero entries.

We define

$$
R_{\mathrm{P} k}=\rho\left(\mathrm{K}_{k k}\right)
$$

as the $R_{0}$-value on patch- $k$. The patch- $R_{0}$ values, $R_{\mathrm{P} k}$, $k=1 \cdots n$ measure the expected number of secondary infections that occur on patch- $k$ only, over the average infectious lifetime of a single primary infective on patch$k$ and assuming the whole system of patches to contain only susceptibles. As such, $R_{\mathrm{P} k} \leqslant R_{0}$, since $R_{0}$ is the expected number of secondary infections for the whole system. Migration between patches will normally mean that it is impossible for the infection to become extinct in one patch while persisting in others, so $R_{\mathrm{P} k}$ does not provide a threshold quantity for persistence in an individual patch. 

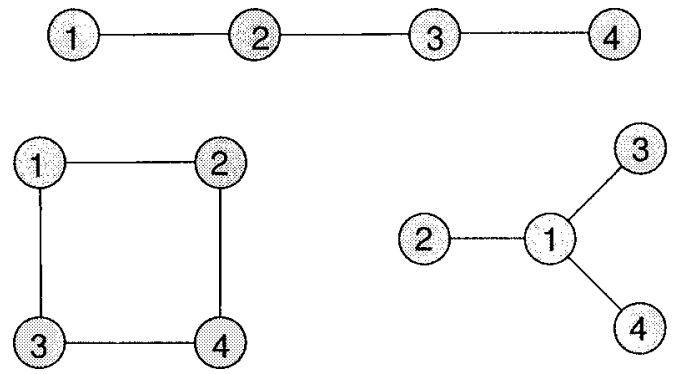

FIG. 2. The spatial configurations of the three generic metapopulation structures with four patches: the chain, loop, and spider.

We also define $R_{\mathrm{M} k}, k=1 \cdots n$, as the expected number of secondary infections in the whole system, due to a single primary infection on patch- $k$,

$$
R_{\mathrm{M} k}=\rho\left(\sum_{l=1}^{n} \mathrm{~K}_{l k}\right)
$$

The overall system $R_{0}$ value lies between the maximum and minimum $R_{\mathrm{M} k}$ values.

We give the structure of matrices $M$, and hence the next generation matrices, for the three metapopulation structures each consisting of four patches (the spider, chain and loop) illustrated in Fig. 2. These configurations represent some generic connections for networks of populations (see Hess, 1996; note that Hess refers to the chain configuration as a necklace configuration).

Spider of 4 patches. Migrations are only possible between the core and the satellites. Hence if patch-1 is the core,

$$
\mathrm{M}=\left(\begin{array}{cccc}
\mathrm{K}_{11} & \mathrm{~K}_{12} & \mathrm{~K}_{13} & \mathrm{~K}_{14} \\
\mathrm{~K}_{21} & \mathrm{~K}_{22} & 0 & 0 \\
\mathrm{~K}_{31} & 0 & \mathrm{~K}_{33} & 0 \\
\mathrm{~K}_{41} & 0 & 0 & \mathrm{~K}_{44}
\end{array}\right) .
$$

Chain of 4 patches. Migrations are possible to neighbouring patches, but there are "end effects," hence

$$
M=\left(\begin{array}{cccc}
\mathrm{K}_{11} & \mathrm{~K}_{12} & 0 & 0 \\
\mathrm{~K}_{21} & \mathrm{~K}_{22} & \mathrm{~K}_{23} & 0 \\
0 & \mathrm{~K}_{32} & \mathrm{~K}_{33} & \mathrm{~K}_{34} \\
0 & 0 & \mathrm{~K}_{43} & \mathrm{~K}_{44}
\end{array}\right)
$$

Loop of 4 patches. Migrations are possible to neighbouring patches, hence

$$
M=\left(\begin{array}{cccc}
\mathrm{K}_{11} & \mathrm{~K}_{12} & 0 & \mathrm{~K}_{14} \\
\mathrm{~K}_{21} & \mathrm{~K}_{22} & \mathrm{~K}_{23} & 0 \\
0 & \mathrm{~K}_{32} & \mathrm{~K}_{33} & \mathrm{~K}_{34} \\
\mathrm{~K}_{41} & 0 & \mathrm{~K}_{43} & \mathrm{~K}_{44}
\end{array}\right) .
$$

\section{A MODEL FOR TUBERCULOSIS IN POSSUMS}

We now apply the general model, defined by Eqs. (1)-(6), to the problem of tuberculosis in possums. The general model allowed for different contact rate functions between classes. Since there are not yet sufficient data for possums available to differentiate contact rates we assume an overall contact function $C$, representing the rate of contacts of one individual with other possums. For example, $C_{12}$ is the rate of adult contacts made by an individual juvenile, so $C_{12}$ is $C$ multiplied by the proportion of the population which are adults, $N_{2 k} / P_{k}$. Thus,

$$
\begin{array}{ll}
C_{11}=C \frac{N_{2 k-1}}{P_{k}}, & C_{12}=C \frac{N_{2 k}}{P_{k}}, \\
C_{21}=C \frac{N_{2 k-1}}{P_{k}}, & C_{22}=C \frac{N_{2 k}}{P_{k}} .
\end{array}
$$

We also let $\beta_{11}=\beta_{12}=\beta_{21}=\beta_{22}$. For the contact rate function we use the form (see Roberts, 1996)

$$
C(P / K)=\frac{c_{0} P / K}{(1-\varepsilon)+\varepsilon P / K},
$$

where $c_{0}$ is the contact rate at carrying capacity $(P=K)$ and the shape parameter $0 \leqslant \varepsilon<1$ governs the degree to which transmission fall between the extremes of frequency dependent transmission $(\varepsilon \rightarrow 1)$ and density dependent transmission $(\varepsilon=0)$. Following Roberts (1996) we take $\varepsilon=0.5$.

In the general model birth and death rates could depend on any local patch demographic parameters; we assume here that $B_{k}=B$ with the function $B$ an increasing function only of the local population density scaled with respect to local carrying capacity $\left(P_{k} / K_{k}\right)$; and $D_{2 k-1}=D_{1}, D_{2 k}=D_{2}$, both decreasing functions of the local scaled population density. For the per-capita birth rate we choose

$$
B(P / K)=\left\{\begin{array}{lll}
b-r \delta(P / K)^{\theta}, & \text { for } & P \leqslant K \\
b_{0} e^{-b_{1}(P / K)^{\theta}}, & \text { for } & P>K,
\end{array}\right.
$$


and for the juvenile and adult per-capita death rates

$$
\begin{aligned}
& D_{1}(P / K)=d_{1}+(1-\zeta) y(P / K)^{\theta} \\
& D_{2}(P / K)=d_{2}+\zeta y(P / K)^{\theta} .
\end{aligned}
$$

Parameters are defined and their default values given in Table I. The carrying capacity $(K)$ refers to the equilibrium population of the patch in isolation. The effect of migration means that the equilibrium population density may be higher or lower than $K$. The exponential form in the birth rate for population densities larger than $K$ is necessary to prevent unrealistic negative values arising in the model, This choice of birth and death rates is an extension of that used in Roberts (1996). Other derived constants $\left(b, b_{0}, b_{1}, y\right)$ are defined below.

The parameter $r$ in Eq. (13) represents the maximum rate of increase of the population, determined as the largest eigenvalue of

$$
\left(\begin{array}{cc}
-\left(d_{1}+a\right) & b \\
a & -d_{2}
\end{array}\right)
$$

(which comes from considering the infection-free population dynamics with no density dependence or migration). Note that, in the limit $a \rightarrow \infty$, corresponding to the time spent as a juvenile tending to zero, $r \rightarrow b-d_{2}$, the value for the non-age-structured population.

The parameters $b_{0}$ and $b_{1}$ in Eq. (13) are chosen so that $B(P)$ is continuous and has continuous first-order derivative at $P=K$. This gives

$$
b_{0}=(b-r \delta) e^{b_{1}}, \quad b_{1}=\frac{r \delta}{b-r \delta} .
$$

The constant $y$ in Eq. (14) is determined from the requirement that, in the absence of any migration, the infection-free equilibrium population is the carrying capacity $K$; a nonzero population density exists provided

$$
\left|\begin{array}{cc}
-\left(d_{1}+(1-\zeta) y+a\right) & b-r \delta \\
a & -\left(d_{1}+\zeta y\right)
\end{array}\right|=0
$$

and $y$ is the positive root of this equation.

In Table I we list parameter values for possum demographics and epidemiology, mostly from Roberts (1996) where their values are discussed in detail.
TABLE I

Possum Demographic and Epidemiological Parameters Used in This Paper

Parameters Symbol Values

density-independent per-capita growth rate $\left(\mathrm{yr}^{-1}\right)$ density-independent per-capita death rates $\left(\mathrm{yr}^{-1}\right)$ logistic shape parameter

density dependence factor: births vs. deaths density dependence factor: juveniles vs. adults carrying capacity (possums ha $\mathrm{h}^{-1}$ ) per-capita maturation rate $\left(\mathrm{yr}^{-1}\right)$ tuberculosis horizontal transmission rate $\left(\mathrm{yr}^{-1}\right)$ tuberculosis per-capita death rate $\left(\mathrm{yr}^{-1}\right)$ per-capita latent-infectious rate $\left(\mathrm{yr}^{-1}\right)$ contact rate factor pseudo-vertical transmission probability probability of migrating from birth-patch migration survival probability

\begin{tabular}{cr}
$r$ & 0.1 \\
$d_{1}, d_{2}$ & 0.17 \\
$\theta$ & 3.0 \\
$\delta$ & 0.5 \\
$\zeta$ & 0.5 \\
$K$ & 10.0 \\
$a$ & 1.0 \\
$\beta_{h} \times c_{0}$ & 5.0 \\
$\alpha$ & 3.0 \\
$\sigma$ & 5.0 \\
$\varepsilon$ & 0.5 \\
$\beta_{v}$ & 1.0 \\
$z$ & 0.5 \\
$s$ & 1.0 \\
\hline
\end{tabular}

Note. These have been sourced from Roberts (1996) with the exception of the following: $\zeta, z$, $s$-these are discussed in the text.

In Roberts (1996), where a non-age-structured model was used, the per-capita birth rate $b$ was defined as the rate of births per possum per year, but here $b$ is the rate per adult per year. For a comparison of the models, we set the density-independent growth rate to be the same in both models $r=0.1 \mathrm{yr}^{-1}$. We then obtain from Eq. (15) $b=0.343 \mathrm{yr}^{-1}$. In the absence of data on the relative effect of density dependence on adults verses juveniles, the parameter $\zeta$ was set to be 0.5 , (compare $\delta=0.5$ in Roberts (1996)). Efford (1998) suggests the value of $z_{l}$ will depend on ecological circumstances. However, we assume a value $z_{l}=z=0.5$, the same for each patch, to obtain an indicative result corresponding to some juveniles migrating out of their birth-patch and some remaining. We also assume that the destinations of migrating juveniles are evenly distributed among patches connected to the birth patch, with a survival rate of $s=1.0$ for juvenile possums migrating along each link, although we also consider $s<1.0$. The package Scilab was used for all numerical calculations.

\section{RESULTS FOR TUBERCULOSIS IN POSSUMS}

We first consider the dynamics in a single homogeneous population so that we can later identify the effects of migration. We then consider a two-patch model and investigate environmental heterogeneity in the form of different carrying capacities and different areas. Finally 

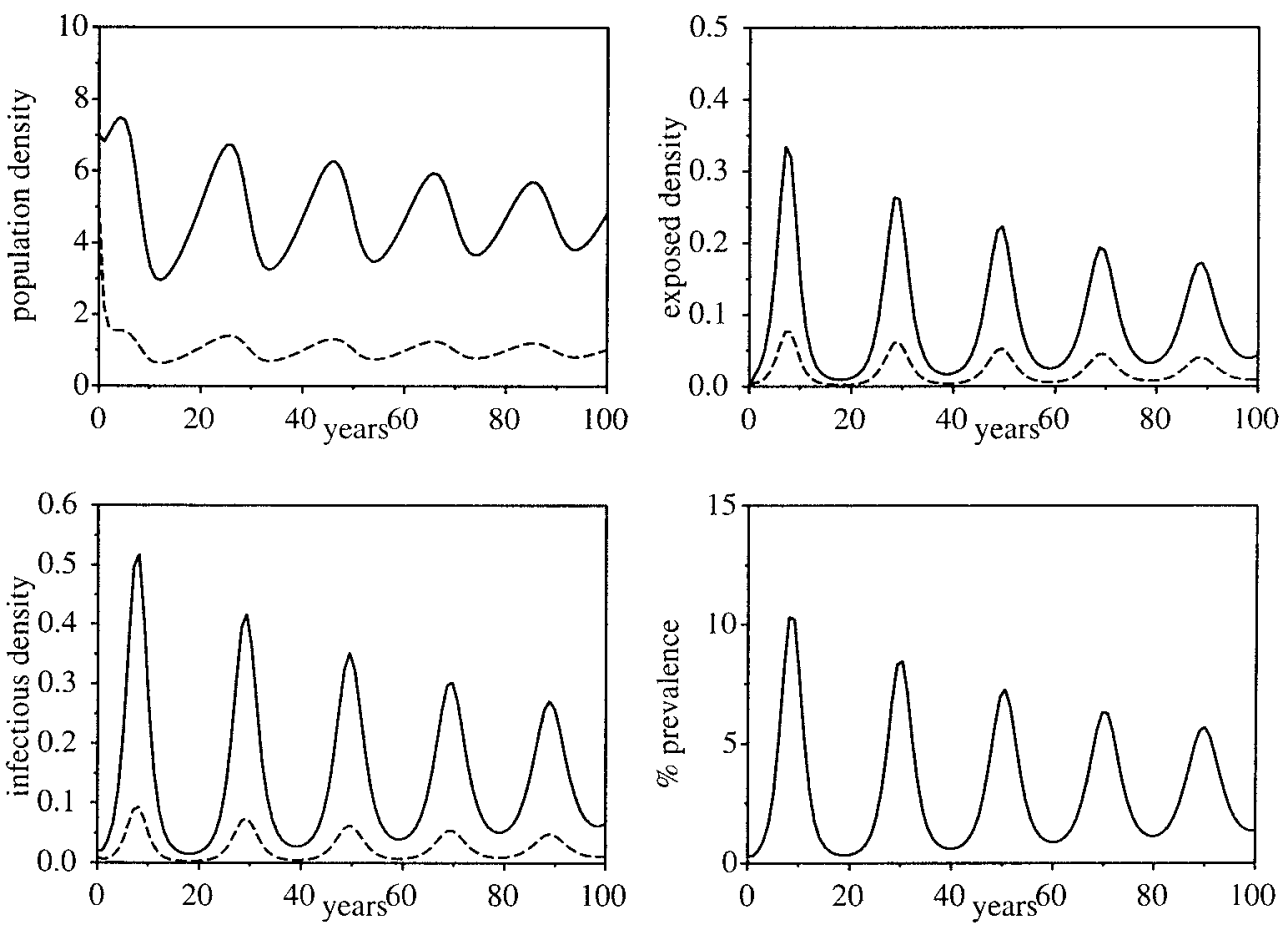

FIG. 3. The density of possums, exposed possums and infectious possums over time on a single patch model. The dashed lines represent the juveniles, the solid lines the total population (adults+juveniles). The graph on the bottom right is the prevalence, which is the ratio of the total number of infectious possum to the total population. Parameter values are from Table I.

we consider three generic network configurations for multiple patches. For each study the initial conditions used were 50 and $20 \%$ of the carrying capacity for the adult and juvenile populations with zero for both exposed classes and $0.1 \%$ of the carrying capacity for the initial densities of juvenile and adult infectives.

\section{A Single Patch}

Figure 3 shows the development of the possum populations and tuberculosis over time for a single homogeneous patch without any migration. The population density, exposed and infectious densities and the prevalence all exhibit weakly damped oscillations. The oscillations in population density lag the infectious density. For this scenario $R_{0}=1.55$.

In comparison with simpler $S I$ and $S E I$ models without age structure (Roberts, 1996), the prevalence peaks are further apart; at approximately 20 years compared to 10-12 years for the SI non-age-structured model. Part of this difference can be attributed to the presence of an exposed class, as modifying the Roberts (1996) model to include an exposed class increases the interval to 15 years. The age-structured model also predicts smaller peaks than the two nonstructured models, but the damping of the peaks is less pronounced.

\section{Two Patches}

In Fig. 4 we plot the population densities and prevalence for a two-patch model where the patches have equal areas but different carrying capacities $\left(K_{1}, K_{2}\right)=$ $(10,8)$ and $\left(K_{1}, K_{2}\right)=(10,1)$. We use this to investigate possible effects of large differences in habitat types.

Migration has a significant effect on the dynamics when the patches have very different no-migration carrying capacities. The population density and prevalence when $K_{2}=8$ are similar to those for the singlepatch model, but those when $K_{2}=1$ are very much different. The damping of the oscillations is greater for a larger difference in carrying capacity. In both cases the prevalences tend to steady-state values; the patches with the lower carrying capacities have the higher steady-state prevalences.

In Table II, $R_{0}$ is calculated as $K_{2}$ varies from 10 down to 1 , while $K_{1}$ remains at 10 . Also shown are the infectionfree equilibria for both patches and $R_{\mathrm{P} 1}, R_{\mathrm{P} 2}, R_{\mathrm{M} 1}$, and $R_{\mathrm{M} 2}$ which provide measures of the transmission potential on an individual patch.

As the difference in migration-free carrying capacity becomes greater $R_{0}$ increases from its minimum value $\left(R_{0}=1.55\right.$ for a single patch or purely symmetrical two-patch model) to $R_{0}=1.67$ for $K_{2}=1$. Thus habitat 

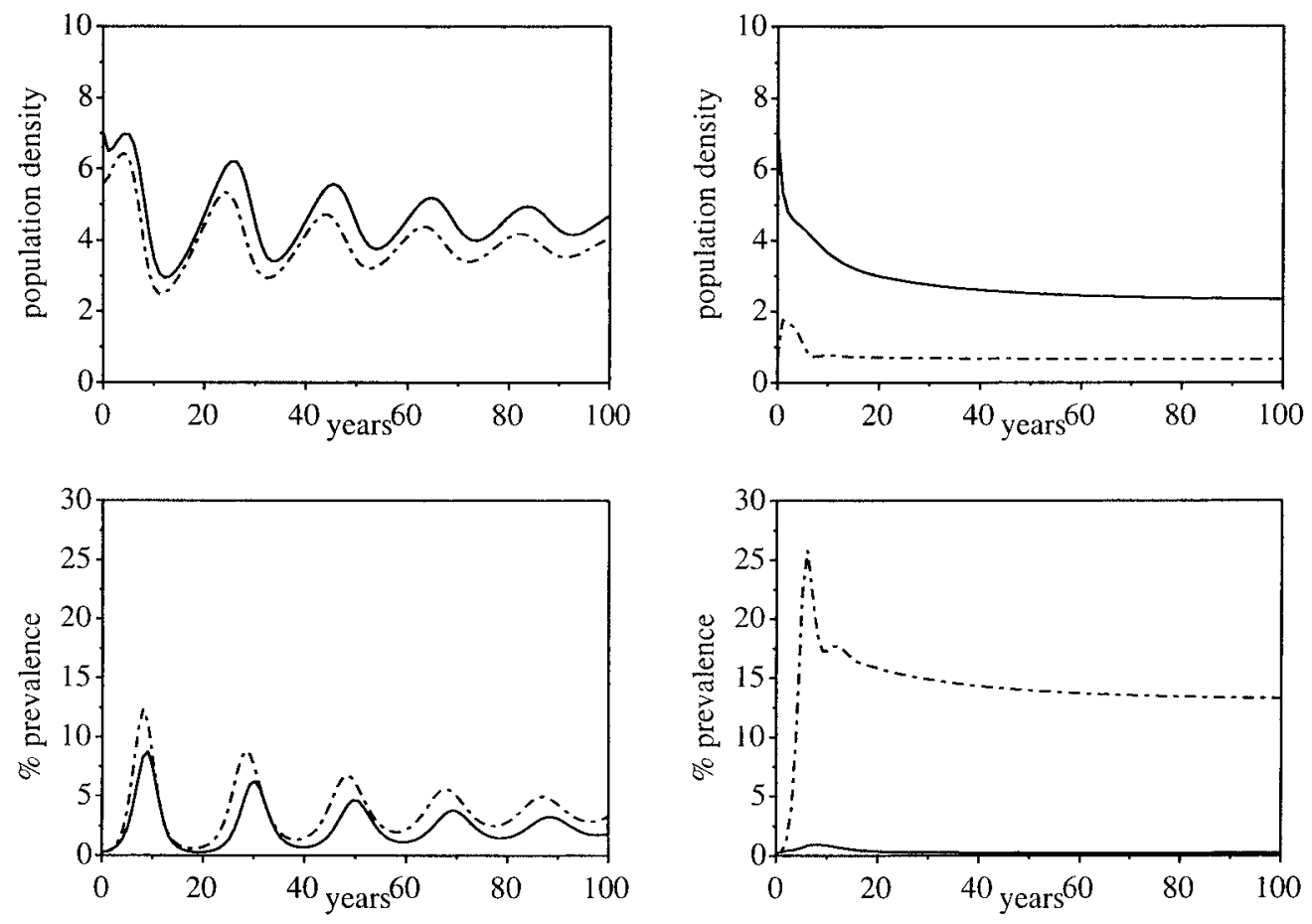

FIG. 4. Population density (sum of adults and juveniles) and prevalence of tuberculosis over time for two patches with equal areas but different carrying capacities. On the left, $\left(K_{1}, K_{2}\right)=(10,8)$ and on the right, $\left(K_{1}, K_{2}\right)=(10,1)$ possums ha ${ }^{-1}$. In each graph patch-1 population density and prevalence is given by a solid line and patch- 2 by a dashed line. Other parameters are from Table I.

differences do not greatly change the overall propensity for the infection to persist in the overall system. However, the distribution of infection between the two patches changes significantly, as seen by the values of the equilibrium prevalence $Z_{1}^{*}$ and $Z_{2}^{*}$.

In Table II, $R_{\mathrm{P} 1}$ and $R_{\mathrm{P} 2}$ are always less than $R_{0}$, whereas $R_{0}$ always lies between $R_{\mathrm{M} 1}$ and $R_{\mathrm{M} 2}$. The larger $R_{\mathrm{M} k}$ and $R_{\mathrm{M} k}$ values coincide with the larger values of the infection-free equilibria. Migration increases the infection-free equilibrium density in patch- 2 when it has a smaller no-migration carrying capacity, and decreases

\section{TABLE II}

Effect of Varying the No-Migration Carrying Capacity of Patch-2 on the Basic Reproduction Ratio $\boldsymbol{R}_{0}$, the Individual Patch Basic Reproduction Ratios $\boldsymbol{R}_{\mathrm{P} 1}, \boldsymbol{R}_{\mathrm{P} 2}, \boldsymbol{R}_{\mathrm{M} 1}, \boldsymbol{R}_{\mathrm{M} 2}$, the Infection-Free Equilibrium Population Densities $P_{1}^{*}, P_{2}^{*}$, and the Equilibrium Percentage Prevalences, $Z_{1}^{*}$ and $Z_{2}^{*}$ for Each Patch of a Two-Patch System

\begin{tabular}{rrrrrrrrrrr}
\hline$K_{1}$ & \multicolumn{1}{c}{$K_{2}$} & $R_{0}$ & $R_{\mathrm{P} 1}$ & $R_{\mathrm{P} 2}$ & $R_{\mathrm{M} 1}$ & $R_{\mathrm{M} 2}$ & $P_{1}^{*}$ & $P_{2}^{*}$ & $Z_{1}^{*}$ & $Z_{2}^{*}$ \\
\hline 10.0 & 10.0 & 1.55 & 1.52 & 1.52 & 1.55 & 1.55 & 10.0 & 10.0 & 3.0 & 3.0 \\
10.0 & 8.0 & 1.56 & 1.49 & 1.55 & 1.52 & 1.58 & 9.4 & 8.5 & 2.4 & 3.7 \\
10.0 & 4.0 & 1.62 & 1.35 & 1.62 & 1.38 & 1.64 & 7.7 & 4.9 & 1.0 & 6.8 \\
10.0 & 2.0 & 1.66 & 1.17 & 1.66 & 1.20 & 1.68 & 5.8 & 2.8 & 0.4 & 10.4 \\
10.0 & 1.0 & 1.67 & 0.93 & 1.67 & 0.95 & 1.69 & 4.0 & 1.5 & 0.2 & 13.1 \\
\hline
\end{tabular}

Note. Parameter values from Table I. the density in patch-1. This has the consequent effect of reducing $R_{\mathrm{P} 1}$ and $R_{\mathrm{M} 1}$ while increasing $R_{\mathrm{P} 2}$ and $R_{\mathrm{M} 2}$ compared to where the patches had the same migration free carrying capacity. We would expect this to happen, as reducing the population density reduces the rate of contacts.

For $K_{2}=1$ the values of $R_{\mathrm{P} 1}$ and $R_{\mathrm{M} 1}$ have fallen below one. From Fig. 4 it might appear that the infection has died out in patch-1 while persisting in patch-2, but this is not the case. The equilibrium prevalence is approximately $0.2 \%$, as calculated numerically from equations (1-6). This raises the question of whether it is ever possible to have complete extinction of the disease on one patch with persistence on the other patch (or patches). From Eq. (5), for a two-patch model, if we set $E_{1}=E_{2}=I_{1}=I_{2}=0$, with $E_{3} \neq 0$, then the equation for $k=1$ simplifies to

$$
\frac{d E_{2}}{d t}=a m_{12} E_{3}>0 \quad \text { if } \quad m_{12} \neq 0
$$

This shows it is impossible to achieve an equilibrium with the infection extinct in patch-1 and persisting in patch-2, provided there is some migration from patch- 2 to patch-1. 


\section{TABLE III}

Effect of Varying the Areas $A_{1}, A_{2}$ of Patches, and the Survival Probabilities $s$ for Migrating Possums, on $R_{0}$ and the Infection-Free Equilibrium Population Densities $P_{1}^{*}, P_{2}^{*}$ of a Two-Patch System

\begin{tabular}{crrrrr}
\hline$s$ & $A_{1}$ & \multicolumn{1}{c}{$A_{2}$} & $R_{0}$ & $P_{1}^{*}$ & $P_{2}^{*}$ \\
\hline 1.0 & 10.0 & 10.0 & 1.55 & 10.0 & 10.0 \\
1.0 & 12.0 & 8.0 & 1.58 & 9.0 & 11.0 \\
1.0 & 14.0 & 6.0 & 1.62 & 7.8 & 12.1 \\
1.0 & 16.0 & 4.0 & 1.65 & 6.4 & 13.4 \\
0.6 & 10.0 & 10.0 & 1.42 & 8.3 & 8.3 \\
0.6 & 12.0 & 8.0 & 1.47 & 7.4 & 9.2 \\
0.6 & 14.0 & 6.0 & 1.52 & 6.4 & 10.1 \\
0.6 & 16.0 & 4.0 & 1.57 & 5.2 & 11.1 \\
\hline
\end{tabular}

Note. Parameter values from Table I.

In Table III, we examine two patches with equal carrying capacity but different areas. Migration rates between the patches are no longer the same; there is a net movement from the larger patch to the smaller patch (see Eq. (7)). As the difference between patch areas becomes greater, the migration rates between them become less symmetrical. This changes the infection-free equilibrium population densities of the patches; decreasing that of the larger patch and increasing that of the smaller. The effect of reducing the survivability of migrating possums (for example $s=0.6$ ) is to decrease $R_{0}$, mainly due to the loss of susceptibles from the system. The larger the migration mortality $(1-s)$ the smaller is the value of both infection-free equilibria, and consequently, the value of $R_{0}$.

We also examined the sensitivity of the model to the parameter $z$, the probability of a maturing juvenile leaving its birth patch. We set $\left(K_{1}, K_{2}\right)=(10,5)$ and found: for $z=0.5$, the two infection-free equilibrium population densities are $P_{1}^{*}=8.2$ and $P_{2}^{*}=5.9$, with $R_{0}=1.60$; for $z=1$ (all possums migrate) $P_{1}^{*}=6.7$, $P_{2}^{*}=6.0$ and $R_{0}=1.59$; and for $z=0$ (no migration) $P_{1}^{*}=10, P_{2}^{*}=5$ and $R_{0}=1.55$. These results suggest that the infection-free equilibria are quite sensitive to the value of $z$ but $R_{0}$ is not.

\section{Multiple Patches}

For multiple patches we consider the three generic network configurations illustrated in Fig. 2. We compute $R_{0}$ for each configuration for different numbers of patches, with all patches having equal areas and carrying capacities. The results are shown in Fig. 5.

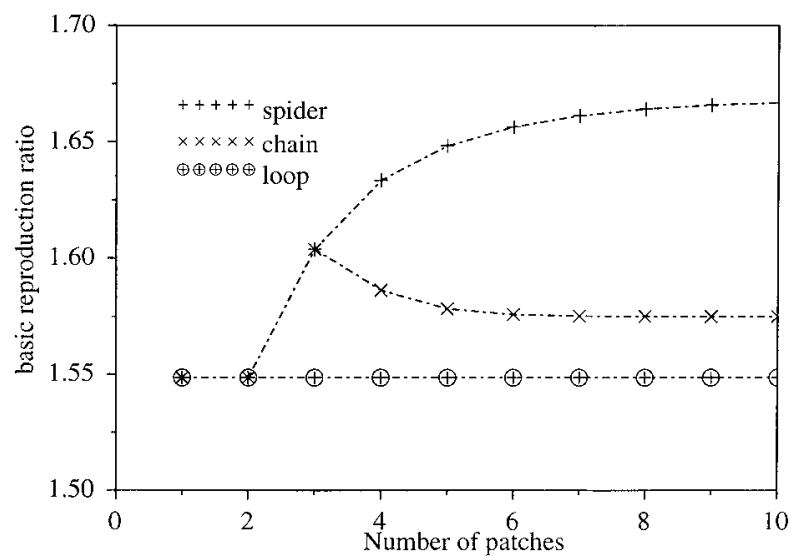

FIG. 5. Comparison of $R_{0}$ vs number of patches for the loop, chain, and spider configurations, with all patches having identical carrying capacity and area. Parameter values are from Table I.

For the loop configuration the value of $R_{0}$ does not change due its symmetry. Clearly the chain and spider configurations are identical for up to three patches. For the parameter values used to construct Fig. 5, the values of $R_{0}$ for the chain and spider configurations asymptote to a limit - effectively 6 patches is equivalent to a larger number of patches. This result would not necessarily be true for nonidentical patches or other sets of parameter values. Also, in obtaining the results presented in Fig. 5, it was assumed that all migration from the outer patches of the chain configuration is to adjacent patches, with no animals migrating out of the system. The patches adjacent to the end patches then have equilibrium diseasefree population densities that exceed $K$, which explains why $R_{0}$ for these configurations is higher than for the loop configuration with the same number of patches. The exploration of results for other sets of parameter values suggests that this may be a general result, but it indicates that $R_{0}$ for the spider configuration may be higher or lower than for the other configurations (results not shown).

\section{CONTROL SCENARIOS FOR TUBERCULOSIS IN POSSUMS}

One of the main applications of models for the spread of tuberculosis in possums is the design of control strategies and buffer regions to prevent, or at least minimise the spread of tuberculosis to farmland. We consider three scenarios each of which is based on one of the generic configurations discussed above:

1. a spider configuration - a forest reserve with links to smaller forested areas on adjacent farmland; 
2. a chain configuration - a forested area along a river;

3. a loop configuration - a forested area surrounding a small village or lake (where possums cannot live).

For each scenario we examine strategies for the eradication of infection. By eradication, we mean that the prevalence not only tends to zero but that if an infected individual is subsequently introduced into the region there will not be a major outbreak $\left(R_{0}<1\right)$.

Control is generally achieved by the distribution of poison baits. Given that resources spent on laying the baits are limited there is considerable interest in the question of how best to distribute them. In particular, is it better to apply control to a subset of patches or to the migration routes between patches. In practice, the cost of distribution is much higher than the cost of the baits, so we assume cost to be proportional to area (see also Kao and Roberts, 1999). Control strategies which can eliminate coverage of some of the areas are therefore worth considering. In the model, control is implemented by increasing the two per-capita death rates $D_{1}$ and $D_{2}$ by the culling rate appropriate for each patch.

\section{Spider Configuration: A Forest Surrounded by Small Outgrowths}

Consider a small forest, of area 1000 ha, referred to as patch-1, surrounded by farmland which has 10 habitat areas (patches 2-11) scattered on it, each of area 100 ha. Each of the smaller patches is linked to the central patch by a single migration route, with maturing possums migrating in each direction with equal probability. All patches have a no-migration carrying capacity of 10 possums $\mathrm{ha}^{-1}$. We assume that migration only occurs between the forest and farmland patches and not between the separate farmland patches.

For this scenario with no control applied $R_{0}=1.55$; $R_{\mathrm{P} k}=1.52$ (equal for all patches) and $R_{\mathrm{M} k}=1.55=R_{0}$ for all patches. This suggests that patch-1 and patches 2-11 will require similar control efforts. We consider control of the forest only, control of all of the surrounding patches on the farmland, control of the whole system, and control of the links to and from the forest. Results for these strategies are shown in Fig. 6.

The results for controlling the forest only and for controlling the surrounding farmland only are the same, with a steady decrease in the value of $R_{0}$ as the per-capita culling rate is increased. The critical culling rate (where $\left.R_{0}=1\right)$ is approximately $0.42 \mathrm{yr}^{-1}$. After $0.5 \mathrm{yr}^{-1}$ the value of $R_{0}$ drops more quickly; at approximately $0.55 \mathrm{yr}^{-1}$ the infection-free population density for the

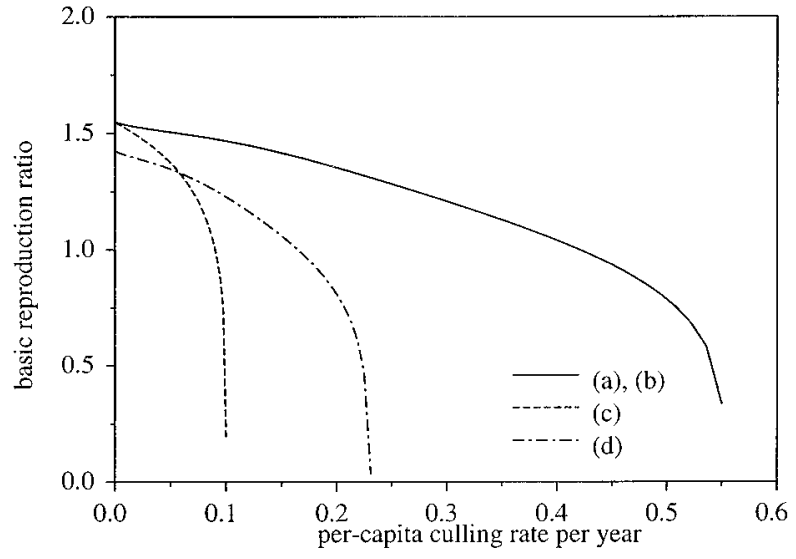

FIG. 6. Spider configuration: central forest-patch surrounded by 10 small farmland patches with migration only between each farmland patch and the forest patch. Plot of $R_{0}$ vs culling rate, for (a) control in patch-1 only (the forest); (b) control applied to patches 2-11 (farmland)-identical to control applied to patch only; (c) control applied to all patches; and (d) control applied to patch-1 only with $1-s=0.4$ mortality on all the migration routes. Parameter values are from Table I.

whole system is approaching zero, however, such high culling rates would be difficult to achieve in practice. In contrast, the decrease in $R_{0}$ for controlling all 11 of the patches is much more rapid. Here the critical culling rate is approximately $0.09 \mathrm{yr}^{-1}$, which is similar to the value obtained by Roberts (1996), for a single-patch SI model with the same parameter values and no age-structure.

These critical values for control are sensitive to the density-independent growth rate $r$. Doubling $r$ approximately doubles the critical value of the culling rate for controlling every patch. For controlling just patch-1, doubling $r$ does not give a critical value: $R_{0}$ then asymptotes to approximately 1.2 as the culling rate increases, i.e., for sufficiently high $r$ it is impossible to reduce $R_{0}$ for the system below 1 by controlling the central patch only.

This scenario contains a large degree of symmetry. Because all the outer patches have the same area and carrying capacities, with no migration between them, it is equivalent to a two-patch system where both patches are equal. This explains why controlling the outer patches produces the same results as controlling only the inner patch. If we destroy the symmetry by making the carrying capacity of the outer patches different then control effectiveness depends on the individual carrying capacities. For example, if we take $K_{1}=10, K_{i}=i$, for $i=$ $2 \cdots 11$, we obtain $R_{\mathrm{P} 1}=1.45$, and $R_{\mathrm{P} k}$, for $k=2 \cdots 11$, ranging from 1.67 for patch-2 to 1.49 for patch-11. Similarly, $R_{\mathrm{M} 1}=1.48, R_{\mathrm{M} 2}=1.69$ and $R_{\mathrm{M} 11}=1.52$. This suggests it will be better to control patches $2-11$ than it would to control only patch-1. Indeed this is the case with 
the critical culling rate (where $R_{0}=1$ ) approximately $0.41 \mathrm{yr}^{-1}$ for controlling patches $2-11$ and $>0.5 \mathrm{yr}^{-1}$ for controlling only patch-1. Thus the values $R_{\mathrm{M} k}$ and $R_{\mathrm{P} k}$ seem to provide useful indicators as to where control should be focused.

Another alternative is to control the links by setting up a buffer zone between the forest and farmland patches and carrying out control operations in the buffer zone as well as the forest. Here we assume a fraction $1-s$ of migrating possums are killed by poison baits in the buffer zone. Results for this strategy are also shown in Fig. 6 where the critical value of the culling rate is approximately $0.17 \mathrm{yr}^{-1}$.

\section{Chain Configuration: Along a River}

In this scenario, we suppose there are 11 forested patches along a river, labelled 1 to 11 , respectively. We assume the carrying capacity and area for each patch to be identical.

When no control is applied $R_{0}=1.57, R_{\mathrm{P} 1}=R_{\mathrm{P} 11}=$ $1.40, R_{\mathrm{P} 2}=R_{\mathrm{P} 10}=1.57$ with $R_{\mathrm{P} k}=1.52$ for $k=3 \cdots 9$ (not all exactly equal). Similarly, $R_{\mathrm{M} 1}=R_{\mathrm{M} 11}=1.41$, $R_{\mathrm{M} 2}=R_{\mathrm{M} 10}=1.61$ with $R_{\mathrm{M} k}=1.55$ for $k=3 \cdots 9$. These values suggest that patches 2 and 10 might require greater control effort.

We compare control applied to patch-1 only, control applied to every second patch, and control applied to all patches. We also consider the effect of control applied to patch-1 with increased mortality of dispersing juvenile possums. The results are given in Fig. 7.

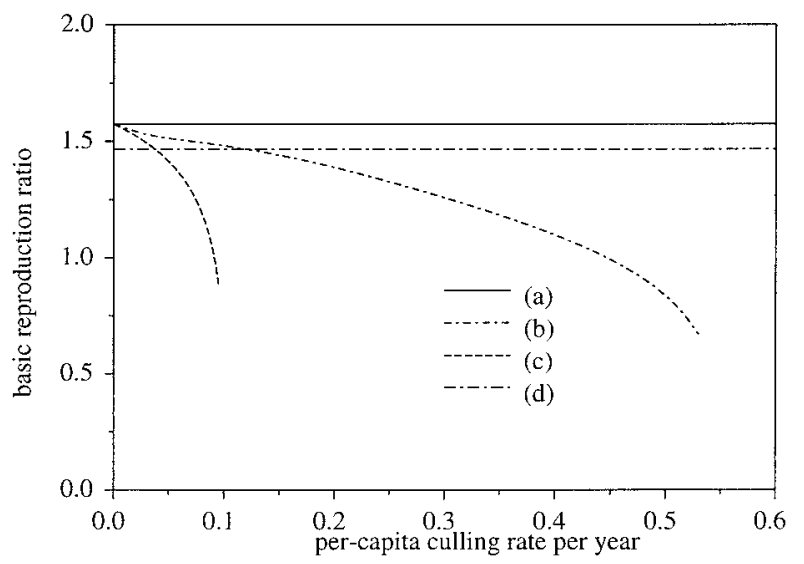

FIG. 7. Chain configuration: along a river. $R_{0}$ vs culling rate for (a) control applied to patch-1 only (end patch); (b) control applied to every second patch starting from patch-2; (c) control applied to all patches; and (d) control applied to patch-1 only with $1-s=0.4$ mortality along all the migration routes. Parameter values are from Table I.
When only patch-1 is controlled the value of $R_{0}$ does not change (even for much higher per-capita culling rates than those illustrated in Fig. 7), showing that it is impossible to eradicate the disease from the system by this method. Plotting the infection-free equilibrium population density for each patch against culling rate reveals that the density for patch-1 goes to zero while that for the other patches remains constant as culling rate increases (results not shown). The results for applying control to patch-1 only and to patches $1-5$ are indistinguishable - there is little change in the value of $R_{0}$. This is because the infection persists in the uncontrolled patches (in particular, patch-10 consistently has the largest $R_{\mathrm{P} k}$ and $R_{\mathrm{M} k}$ ) and the infection spreads by migration to the controlled patches. The prevalence is lower in the controlled patches but tuberculosis is not eradicated by this strategy.

By applying control to every second patch eradication of tuberculosis is theoretically possible, with a decrease in $R_{0}$ from 1.57 to below one, for a per-capita culling rate of approximately $0.45 \mathrm{yr}^{-1}$. Controlling every second patch restricts the spread of infection to other patches and thus it is more difficult for it to persist overall. However, these conclusions could be different if we allowed for maturing juveniles to migrate past adjacent patches, this will be considered in future studies.

We also considered the effect of culling patch-1 together with a mortality of $40 \%$ along all migration routes. Although $R_{0}$ is reduced from about 1.55 down to 1.47 , increasing the culling rate on patch-1 does not decrease $R_{0}$. A higher value of migration mortality can reduce $R_{0}$ to below 1 .

\section{Loop Configuration: Around a Small Town or Lake}

Consider 12 identical habitat patches surrounding some obstacle (such as a small town or lake) into which possums cannot move. This is an example of a loop configuration. For this scenario $R_{0}=1.55, R_{\mathrm{P} k}=1.52$, and $R_{\mathrm{M} k}=1.55=R_{0}$; all identical due to symmetry.

The results for 4 different control strategies are shown in Fig. 8: applying control to patch-1 only; to every second patch; to all the patches; and to patch-1 with increased mortality of dispersing juveniles.

Applying control to patch-1 only has almost no effect and an almost identical graph is obtained for applying control to 5 adjacent patches. Applying control to every second patch is more effective, but less effective than applying control to all patches; the same conclusion as that for the chain configuration. We also considered the effect of some patches being missed out, i.e., every patch 


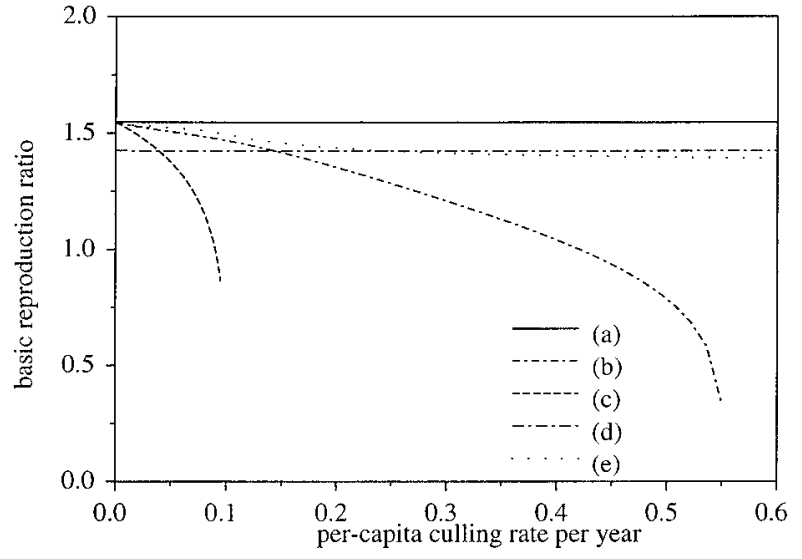

FIG. 8. Loop configuration: around a lake. $R_{0}$ vs culling rate for (a) control applied to patch-1 only; (b) control applied to every second patch; (c) control applied to all patches; (d) control applied to all patches except patches 1 and 12; and (e) control applied to patch-1 only with $1-s=0.4$ mortality on all migration routes. Parameter values are from Table I.

is controlled except for patches 1 and 12 . When only one patch is not controlled the critical value of the culling rate for eradication of the disease increases from approximately 0.09 to 0.22 . However, when two adjacent patches are not controlled, we see from Fig. 8 that the curve appears to asymptote to a value above one which suggests that eradication cannot be achieved.

For the loop configuration we might have expected that applying sufficiently high control to patch-1 would effectively "wipe out" the population in patch-1 and thus the loop configuration would resemble the chain configuration. However, high culling rates applied to patch-1 in the loop configuration do not give exactly the same value of $R_{0}$ as no culling in the chain configuration (1.51 vs 1.54). The reason for this small difference is that, for the chain configuration, there is no migration of possums from the two end patches, 1 and 12, to the surrounding farmland, whereas for the loop configuration, with high cull rates applied to patch-1, there is migration of possums from patches 2 and 12 and these are lost from the system.

\section{DISCUSSION}

We have developed a metapopulation model for the spread of an infectious disease by the migration of exposed juvenile hosts. The model features two age classes - adult and juvenile, and three epidemiological classes - susceptible, exposed (infected but not infectious), and infectious. The model incorporates a variable population size on each patch, with birth, death, and infectious contact rates depending on the local population densities. Births are assumed to be continuous throughout the year. Roberts and Kao (1998) considered a model with discrete birth pulses, butfound the differences between it and a model with continuous births to be small for the parameters appropriate for possum populations, which are the motivation for this study. We have included (pseudo)vertical transmission of infection from mother to offspring, which has been reported to be common for tuberculosis in possums (Morris and Pfeiffer, 1995), although Roberts (1996) found its effect on disease dynamics to be negligible.

We have applied the model to the dynamics of tuberculosis in possums and provided a methodology for computing the basic reproduction ratio for the infection $\left(R_{0}\right)$. The structure of the next generation matrix used to calculate $R_{0}$ features the separation of the network topology, the demographics of the host population, and the epidemiology of the disease; it is easily generalised to large and complex situations.

Three scenarios were considered as examples and used to demonstrate the potential for this model to be developed into a management tool for the control of tuberculosis.

Our model applied to a single patch exhibited weakly damped oscillations in the prevalence of the disease, which were not previously observed in the non-agestructured SI model. Oscillations in prevalence (damped or otherwise) have not, as yet, been reported in possums; data collected over at least 40-60 years or more would be needed. However, they have been reported in badgers (Cheesman et al., 1989). The introduction of a spatial component, through the presence of more than one habitat patch, changes this behaviour dramatically provided there is some asymmetry in the system. For two patches we observed a rapid increase in prevalence, far exceeding that for a single patch, followed by very heavy damping. As the populations in both patches increase due to births, asymmetry in migration between the patches causes a peak in the population density (see Fig. 4). This in turn drives an increase in the density of infected hosts.

The presence of heterogeneity caused only a small increase in the value of $R_{0}$ for tuberculosis in possums. From Table II a 10-fold proportional difference between patch migration-free carrying capacities results in a less than $8 \%$ increase in $R_{0}$. A similar increase was noted in Table III for a 10 -fold difference between patch areas. Inclusion of $40 \%$ mortality of migrating possums resulted in less than a $3 \%$ increase in $R_{0}$.

We have also introduced quantities $R_{\mathrm{P} k}$ and $R_{\mathrm{M} k}$ which are reproduction ratios for individual patches. The $R_{\mathrm{P} k}$ represent the expected number of secondary infections 
on the same patch as a single primary infection, over the average lifetime of an infection. Unlike $R_{0}$, the $R_{\mathrm{P} k}$ do not provide threshold values for infection extinction on a patch, as the disease always persists due to the migration of exposed juveniles from other infected patches. From Table II we see that a higher value of $R_{\mathrm{P} k}$ does correspond to higher values of the equilibrium prevalence. This could be useful as an indicator for devising cost efficient control strategies - areas with higher values of $R_{\mathrm{P} k}$ might be candidates for increased focus of control effort. The $R_{\mathrm{M} k}$ represent the expected number of secondary infections in the whole system due to a single primary infection introduced on patch- $k$. As the quantities $R_{\mathrm{M} k}$ include the effect of migration of exposed possums between patches, they may provide useful indicators of which patches make a more significant contribution to the overall system $R_{0}$ rather than the $R_{\mathrm{P} k}$.

It is useful to think of migration as having two contributions to the system $R_{0}$ value. There is a continuous source of infection through migration of exposed individuals, and the migration of susceptibles can change the infection-free equilibrium population densities from their migration-free values. This second contribution may result in changed values of $R_{\mathrm{P} k}, k=1 \cdots n$, as patches with higher infection-free equilibrium population densities have higher values of $R_{\mathrm{P} k}$, if everything else is equal. The overall system $R_{0}$ is always greater than any of the $R_{\mathrm{P} k}$ values, so the net differential in migration rates of healthy susceptibles between any patches will increase the value of the system $R_{0}$. For the parameters used in this study this second mechanism is more important in determining the value of $R_{0}$.

Figures 6, 7, and 8 show the effects of different control strategies for three spatial configurations of habitat patches. We found that, when applying control equally to all patches, the critical value of the per-capita culling rate was similar to that predicted by a non-age-structured single-patch SI model, as in Roberts (1996). These critical values are sensitive to demographic parameters (see also Roberts (1996)). In the field there is variability in population growth parameters, in particular the density independent birth and death rates $\left(b, d_{1}, d_{2}\right)$ depend on climate and habitat. We have used a density-independent growth rate $r=0.1 \mathrm{yr}^{-1}$, for comparison with Roberts (1996), but this value could be higher (for example Barlow (1991) uses $r=0.2 \mathrm{yr}^{-1}$ ). The implications of this for tuberculosis control are that higher culling rates would be necessary to reduce $R_{0}$ below 1 than have been obtained here. A full systematic, sensitivity analysis of this model, such as that done by Roberts (1996), will be carried out in a future study, where this model will be applied to a specific field control situation.
Of the three network configurations considered here, controlling just a single patch was ineffective for the chain and loop configurations, but could work for the spider configuration provided it is the inner patch which is controlled. This may be an effective strategy if the cost of control is governed mainly by area and the culling rates needed to eradicate the disease can be achieved. For each configuration the effort directed at controlling the patch is offset by the immigration of possums from neighbouring patches. In the case of the spider configuration some progress can be made due to the larger number of connections to the patch being controlled, whereas the failure in the chain and loop configurations are due to the controlled patch only having one or two links to the uncontrolled patches.

We also looked at the effect of controlling a subset of patches. For the chain and loop configurations there was no advantage in controlling half the number of patches if they were adjacent, but there was some advantage in controlling every second patch. Although this was not as effective as controlling every patch, in terms of reduction of $R_{0}$ for a given culling effort, it could be more cost effective. Controlling every second patch makes it more difficult for the infection to establish itself, whereas controlling adjacent patches (while still leaving half the number of patches uncontrolled) leaves two or more patches which can sustain themselves through migration.

We have shown that migration has an important effect on maintaining the infection in a population. Controlling patch-1 while at the same time having $40 \%$ mortality on all the migration links leads to more effective control of the disease (Figures 6, 7, and 8).

One of the potential uses of a spatial model for possum tuberculosis is to explain the evolution of spatial patchiness in prevalence. Patchiness has been observed in a number of field studies (for example, Coleman et al., 1999; Hickling, 1995; Caley et al., 1999), but not in others (Coleman et al., 1994). The results presented in Fig. 4 suggests significant inhomogeneity in prevalence can arise from inhomogeneity in landscape, as suggested by Barlow (1994), especially over a short time scale (say 5-10 years). This may be a contributing factor for observed "hot spots" of infection.

The model developed here is one of the simplest possible models for spatial spread of an infectious agent, yet it still encompasses key biological, epidemiological and topographical features. The behaviour of the model is quite complex even with a relatively small number of patches and a small degree of habitat variation. Ongoing work will extend this model to more complex spatial landscapes and use it to determine optimum strategies for the control of possums and tuberculosis. 


\section{ACKNOWLEDGMENTS}

This study was funded by the Foundation for Research, Science and Technology, contract number C10 420. M.G.R. visited Wageningen with financial assistance from GLW-DLO. We thank Phil Cowan, Peter Caley, and Dave Ramsey for useful discussions, and Lydia van Dijk for help on Scilab programming. We also thank Rowland Kao for some initial work on this problem.

\section{REFERENCES}

Anderson, R. M., and May, R. M. 1991. "Infectious Diseases of Humans: Dynamics and Control," Oxford University Press, New York.

Barlow, N. D. 1991. A spatially aggregated disease/host model for bovine $\mathrm{Tb}$ in New Zealand possum populations, J. Appl. Ecol. 28, 777-793.

Barlow, N. D. 1993. A model for the spread of bovine Tb in New Zealand possum populations, J. Appl. Ecol. 30, 156-164.

Barlow, N. D. 1994. Bovine tuberculosis in New Zealand: Epidemiology and models, Trends Microbiol. 2, 119-125.

Barlow, N. D. 1996. The ecology of wildlife disease control: Simple models revisited, J. Appl. Ecol. 33, 303-314.

Bolker, B. M. 1995. Group report: Spatial dynamics of infectious diseases in natural populations, in "Ecology of Infectious Diseases in Natural Populations" (B. T. Grenfell and A. P. Dobson, Eds.), pp. 384 -398, Cambridge University Press, Cambridge, UK.

Caley, P., Hickling, G. J., Cowan, P., and Pfeiffer, D. U. 1999. Effects of sustained control of brushtail possums on levels of Mycobacterium bovis infection in cattle and brushtail possum populations from Hohotaka, New Zealand, NZ Vet. J. 47, 133-142.

Caley, P., Spencer, N. J., Cole, R. A., and Efford, M. G. 1998. The effect of manipulating population density on the probability of densharing among common brushtail possums, and the implications for transmission of bovine tuberculosis, Wildlife Res. 25, 383-392.

Cheesman, C. L., Wiseman, J. W., and Stuart, F. A. 1989. Tuberculosis: The disease and epidemiology in the badger, a review, Epidem. Inf. 103, 113-125.

Coleman, J. D., Cooke, M. M., Jackson, R., and Webster, R. 1999. Temporal patterns in bovine tuberculosis in a brushtail possum population contiguous with infected cattle in the Ahaura valley, Westland, NZ Vet. J. 47, 119-124.

Coleman, J. D., Jackson, R., Cooke, M. M., and Grueber, L. 1994. Prevalence and spatial distribution of bovine tuberculosis in brushtail possums on a forest-scrub margin, NZ Vet. J. 42, 128-132.

Cowan, P. E. 1990. Brushtail possum, in "The Handbook of New Zealand Mammals” (C. M. King, Ed.), pp. 68-98, Oxford University Press, Oxford.

Diekmann, O., and Heesterbeek, J. A. P. 2000. "Mathematical Epidemiology of Infectious Diseases: Model Building, Analysis and Interpretation," Wiley Series in Mathematical and Computational Biology, Wiley, Chichester.

Diekmann, O., Heesterbeek, J. A. P., and Metz, J. A. J. 1990. On the definition and computation of the basic reproduction ratio $R_{0}$ in models for infectious diseases in heterogeneous populations, J. Math. Biol. 28, 365-382.

Dobson, A. P., and Grenfell, B. T. 1995. Introduction, in "Ecology of
Infectious Diseases in Natural Populations" (B. T. Grenfell and A. P. Dobson, Eds.), pp. 1-19, Cambridge University Press, Cambridge, UK.

Efford, M., 1998. Demographic consequences of sex-biased dispersal in a population of brushtail possums, J. Anim. Ecol. 67, 503-517.

Hanski, I. A., and Gilpin, M. E. 1997. "Metapopulation Biology," Academic Press, San Diego.

Heesterbeek, J. A. P., and Roberts, M. G. 1995. Mathematical models for microparasites of wildlife, in "Ecology of Infectious Diseases in Natural Populations" (B. T. Grenfell and A. P. Dobson, Eds.), p. 90-122, Cambridge University Press, Cambridge, UK.

Hess, G. 1996. Disease in metapopulation models: Implications for conservation, Ecology 77, 1617-1632.

Hickling, G. 1995. Clustering of tuberculosis infection in brushtail possum populations: Implications for epidemiological simulation models, in "Tuberculosis in Wildlife and Domestic Animals" (F. Griffin and G. de Lisle, Eds.), pp. 174-177, University of Otago Press, Dunedin.

Jackson, R. S., Cooke, M. M., Coleman, J. D., Morris, R. S., de Lisle, G. W., and Yates, G. F. 1995. Naturally occurring tuberculosis caused by Mycobacterium bovis in brushtail possums (Trichosurus vulpecula): III. Routes of infection and excretion, NZ Vet. J. 43, 322-327.

Jacquez, J., Simon, C., and Koopman, J. 1995. Core groups and the $R_{0} \mathrm{~s}$ for subgroups in heterogeneous SIS and SI models, in "Epidemic Models: Their Structure and Data" (D. Mollison, Ed.), pp. 279-301, Cambridge University Press, Cambridge, UK.

Kao, R. R., and Roberts, M. G. 1999. A comparison of wildlife control and cattle vaccination as methods for the control of bovine tuberculosis, Epidem. Inf. 122, 505-519.

Lloyd, A. L., and May, R. M. 1996. Spatial heterogeneity in epidemic models, J. Theor. Biol. 179, 1-11.

Louie, K., Roberts, M. G., and Wake, G. C. 1993. Thresholds and stability analysis of models for the spatial spread of a fatal disease, IMA J. Math. Appl. Med. Biol. 10, 207-226.

Mollison, D., and Levin, S. A. 1995. Spatial dynamics of parasitism, in "Ecology of Infectious Diseases in Natural Populations" (B. T. Grenfell and A. P. Dobson, Eds.), pp. 384-398, Cambridge University Press, Cambridge, UK.

Morris, R. S., and Pfeiffer, D. U. 1995. Directions and issues in bovine tuberculosis control in New Zealand, N.Z. Vet J. 43, 256-265.

Murray, J. D. 1990. “Mathematical Biology," Springer-Verlag, New York.

Pech, R. P., and McIlroy, J. C. 1990. A model of the velocity of advance of foot and mouth disease in feral pigs, J. Appl. Ecol. 27, 635-650.

Pfeiffer, D. U. 1994. "The Role of a Wildlife in the Epidemiology of Bovine Tuberculosis," Ph.D. thesis, Massey University.

Roberts, M. G. 1996. The dynamics of bovine tuberculosis in possum populations, and its eradication or control by culling or vaccinations, J. Anim. Ecol. 65, 451-464.

Roberts, M. G., and Kao, R. R. 1998. The dynamics of an infectious disease in a population with birth pulses, Math. Biosc. 149, 23-36.

Ruxton, G. D. 1996. The effects of stochasticity and seasonality on model dynamics: Bovine tuberculosis in badgers, J. Anim. Ecol. 65, 495-500.

Swinton, J., Tuyttens, F., Macdonald, D., Nokes, D. J., Cheeseman, C. L., and Clifton-Hadeley, R. 1997. A comparison of fertility control and lethal control of bovine tuberculosis in badgers: The impact of perturbation induced transmission, Phil. Trans. R. Soc. London B 352, 619-631. 